\title{
Dynamics modeling and nonlinear observer design for vehicle rollover avoidance
}

\author{
Chang-Fang Chen ${ }^{\dagger}$, Ming-Lei Shu ${ }^{\mathrm{a}}$, Ming Yang and Nuo Wei \\ Shandong Computer Science Center (National Supercomputer Center in Jinan) \\ Shandong Provincial Key Laboratory of Computer Networks \\ Jinan 250014, China \\ †e-mail: chenchangfang012@163.com; ${ }^{a} e$-mail: shuml@sdas.org
}

\begin{abstract}
A real-time accurate detecting the risk of an impending vehicle rollover is essential for active rollover avoidance systems. To this end, a 4DOF nonlinear vehicle roll dynamics model is developed, which fully exploit the coupling effects between the yaw dynamics, lateral dynamics, and roll dynamics. An asymptotically stable observer is presented by using the mean value theorem of vectorial form, and the observer gain is derived based on linear matrix inequalities (LMIs). The simulations demonstrate that the developed nonlinear observer can provide reliable estimation of the vehicle states, even under aggressive driving conditions.
\end{abstract}

Keywords: Rollover prevention; Vehicle dynamics; Roll angle; Nonlinear observer; Asymptotically stable; Linear matrix inequalities

\section{Nomenclature}

$C G \quad$ Center of gravity.

$v_{x}, v_{y} \quad$ Longitudinal/lateral velocity.

$m_{\mathrm{u}}, m_{s} \quad$ Vehicle unsprung mass and sprung mass.

$a_{y u}, a_{y s}$ Lateral accelerations of the unsprung mass and sprung mass.

$C_{r}, C_{f}$ Cornering stiffness coefficients of rear and front tires.

$F_{y r}, F_{y f}$ Lateral forces of rear and front tires.

$\alpha_{r}, \alpha_{f}$ Sideslip angles of rear and front tires.

$I_{x x}, I_{y y}, I_{z z}$ Moments of inertia about $\mathrm{x} / \mathrm{y} / \mathrm{z}$ axis.

$l_{r}, l_{f} \quad$ Distances from rear and front wheel axes to $C G$.

$\phi, \dot{\phi} \quad$ Roll angle/roll rate.

$h_{s} \quad$ Distance from CG to roll center.

$\gamma \quad$ Yaw rate 
$\delta_{f} \quad$ Steering angles of front wheels.

$\kappa_{f}, \kappa_{r} \quad$ Front and rear roll-steer coefficients.

$\kappa_{\phi} \quad$ Roll stiffness.

$c_{\phi} \quad$ Roll-damping coefficient

\section{Introduction}

Vehicle rollover is regarded as the vehicle collision type, which has the highest mortality. The statistical results of the National Highway Traffic Safety Administration (NHTSA) show that approximately 3\% of vehicle collisions involve rollover incidents annually, and $33 \%$ of death related to all vehicle collisions are caused by vehicle rollovers [1]. With high- $C G$ vehicles popular, for example, trucks and SUVs, the rollover avoidance systems are further developed because these kinds of vehicles are most likely to be related to vehicle rollover accidents.

To solve this common safety problem, it is needed to detect impending vehicle rollover timely, which can be alleviated by utilizing chassis control units depending on different rollover conditions [2]-[3]. Vehicle rollover prevention systems rely crucially on timely determination of the rollover conditions. To determine an impending rollover timely and accurately, the roll rate and roll angle measurements are necessary. For example, in [4], Ford develops an estimation actuator dependent on the measured roll rate to detect and to avoid impending rollovers. A differential braking based rollover prevention system is proposed by discovering lateral acceleration or wheel liftoff larger than a predefined threshold [5]-[6]. A three-axis accelerometers is used to estimate the roll angle and roll rate for vehicle rollover avoidance [7]. All the proposed schemes detect an impending rollover events by using some forms of predefined values of roll angle and roll rate measurements. However, most of the modern vehicles have no roll rate or roll angle sensors, so an observer is used to provide both roll rate and roll angle estimation for rollover prevention scheme [8]. Furthermore, when the lateral acceleration is larger, the coupling effects between the roll, lateral, and yaw dynamics will become increasingly significant, which can be utilized to improve control performance [9].

To this end, a nonlinear vehicle model with four degrees of freedom (4DOF) is developed, including the lateral dynamics, yaw dynamics, and roll dynamics with some modifications and improvements with respect to the classic roll dynamics. An asymptotically stable observer is proposed for such nonlinear roll dynamics model with bounded Jacobian matrix, and the observer gain matrix is derived based on linear matrix inequalities. According to the mean value theorem, 
the nonlinear vehicle dynamics can be expressed as the convex combination of some known matrices, and the coefficients are time-varying. The simulations indicate that the proposed nonlinear observer can provide reliable estimation of vehicle states even under aggressive driving conditions.

The paper is organized as follows: the coordinate systems is defined for vehicle dynamics in Section II, and a 4DOF nonlinear vehicle roll dynamics model is derived, which consists of lateral, yaw and roll dynamics. By using the modified mean value theorem, an asymptotically stable observer is presented for the nonlinear roll dynamics, and the stability properties are also analyzed in Section III. The performance evaluation and simulations are presented in Section IV. Finally, our results are concluded in Section V.

\section{Vehicle System Model}

\subsection{Translational motions}

As shown in Fig. 1, define three right-hand coordinate systems to describe the rotational and translational movements of the vehicle unsprung and sprung mass dynamics. $O X Y Z$ is the inertial coordinate system located on ground, and $Z$-axis is upward from the ground. $O_{u} X_{u} Y_{u} Z_{u}$ is the unsprung mass coordinate system, which is located on the vehicle chassis and moves around roll center. $O_{s} X_{s} Y_{s} Z_{s}$ is the sprung mass coordinate system rotating relative to $X_{u}$ with the center of gravity $C G$.

It is assumed that the ground is flat, and the vertical and pitch dynamics of vehicles are ignored. Then the vehicle dynamics with four degrees of freedom are derived. The relative motions between $O_{u} X_{u} Y_{u} Z_{u}$ and $O X Y Z$ include the lateral and longitudinal dynamics, i.e., translational motions along $Y$-axis and $X$-axis, and yaw and roll dynamics, i.e., the rotational motions relative to $Z$ axis and $X$-axis, respectively. Assuming that the bank angle of the ground is small, the unsprung mass angular velocity relative to $O X Y Z$ is given as follows

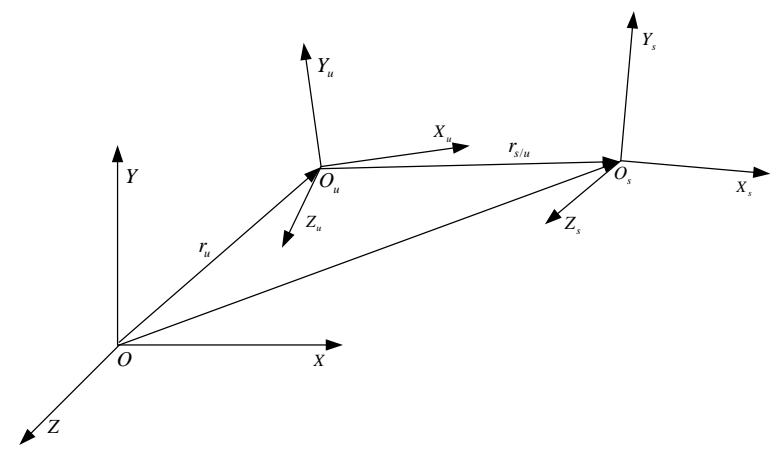

Fig. 1. Reference frames. 


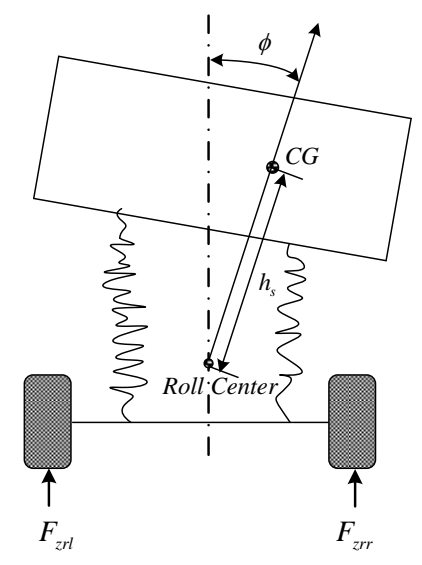

Fig. 2. Geometry of the vehicle.

$$
\Omega_{u}=\left[\begin{array}{lll}
0 & 0 & \gamma
\end{array}\right]^{T}
$$

where the angular velocity of $O_{u}$, i.e., $\Omega_{u}$, is defined in the inertial coordinate system. By neglecting the vertical movement, the unsprung mass acceleration relative to $O_{u} X_{u} Y_{u} Z_{u}$ is expressed as

$$
\begin{gathered}
\ddot{\mathbf{r}}_{u}=\frac{\partial \dot{\mathbf{r}}_{u}}{\partial t}+\Omega_{u} \times \dot{\mathbf{r}}_{u} \\
=\left[\begin{array}{lll}
\dot{v}_{x}-v_{y} \gamma & \dot{v}_{y}+v_{x} \gamma & 0
\end{array}\right]^{T}
\end{gathered}
$$

where $\partial \dot{\mathbf{r}}_{u} / \partial t$ is the time derivative of $\dot{\mathbf{r}}_{u}$ in the rotating coordinate system, i.e., $O_{u} X_{u} Y_{u} Z_{u}$.

From Fig. 2, by ignoring the vertical and pitch dynamics, i.e., movements along $Z$-axis and $Y$-axis, the sprung mass translation motion with respect to $O_{u} X_{u} Y_{u} Z_{u}$ can be evaluated as

$$
\mathbf{r}_{s / u}=\left[\begin{array}{lll}
0 & -h_{s} \sin \phi & 0
\end{array}\right]^{T}
$$

Similar to (2), the acceleration of sprung mass relative to $O_{u} X_{u} Y_{u} Z_{u}$ is as follows

$$
\dot{\mathbf{r}}_{s / u}=\frac{\partial}{\partial t}\left(\mathbf{r}_{s / u}\right)+\Omega_{u} \times \dot{\mathbf{r}}_{s / u}
$$




$$
\begin{aligned}
\ddot{\mathbf{r}}_{s / u} & =\frac{\partial^{2}}{\partial t^{2}}\left(\mathbf{r}_{s / u}\right)+\Omega_{u} \times\left(\Omega_{u} \times \mathbf{r}_{s / u}\right)+2 \Omega_{u} \times \frac{\partial}{\partial t}\left(\mathbf{r}_{s / u}\right) \\
& =\left[\begin{array}{c}
h_{s}(\sin \phi \dot{\gamma}+2 \cos \phi \dot{\phi} \gamma) \\
h_{s}\left(\sin \phi \dot{\phi}^{2}-\cos \phi \ddot{\phi}+\sin \phi \gamma^{2}\right) \\
0
\end{array}\right]
\end{aligned}
$$

Combining (2) and (4), the acceleration of sprung mass relative to the inertia coordinate system $O X Y Z$ is computed as follows

$$
\begin{aligned}
\ddot{\mathbf{r}}_{s} & =\ddot{\mathbf{r}}_{u}+\ddot{\mathbf{r}}_{s / u} \\
& =\left[\begin{array}{c}
\dot{v}_{x}-v_{y} \gamma+h_{s}(\sin \phi \dot{\gamma}+\mathbf{2} \cos \phi \dot{\phi} \gamma) \\
\dot{v}_{y}+v_{x} \gamma+h_{s}\left(\sin \phi \dot{\phi}^{2}-\cos \phi \ddot{\phi}+\sin \phi \gamma^{2}\right) \\
\mathbf{0}
\end{array}\right]
\end{aligned}
$$

\subsection{Rotational Motions}

In an inertial reference frame, the applied torque is equal to the differentiation of angular momentum with respect to time. This rule is generally true, but in solving the movement of a rotating rigid body, it is not effective because the angular velocity of the rotating frame of reference and the moment of inertia tensor will vary with motion. Since the main axes of the moment of inertia tensor are aligned with the coordinate frame located in the rotating body, thus it is needed to change to this frame. In this way, calculations can be simplified due to constant moment of inertia tensor. The inertia tensor $\mathbf{I}_{s}$ around the principal axes of $O_{s} X_{s} Y_{s} Z_{s}$ is given as follows

$$
\mathbf{I}_{s}=\left[\begin{array}{ccc}
I_{x x} & 0 & -I_{x z} \\
0 & I_{y y} & 0 \\
-I_{x z} & 0 & I_{z z}
\end{array}\right]
$$

In the rotating frame of reference, the differentiation of angular momentum with respect to time is

$$
\mathbf{M}=\frac{d}{d t}\left(\mathbf{I}_{u} \Omega_{s}\right)+\Omega_{s} \times\left(\mathbf{I}_{u} \Omega_{s}\right)
$$

where $\Omega_{s}$ represents the angular velocity of the sprung mass relative to $O_{u} X_{u} Y_{u} Z_{u}$ with neglecting the pitch movement. Using the transformation matrix $\mathbf{R}_{t}$ between the coordination systems $O_{u} X_{u} Y_{u} Z_{u}$ and $O_{s} X_{s} Y_{s} Z_{s}$, the inertia tensor $\mathbf{I}_{u}$ in the $O_{u} X_{u} Y_{u} Z_{u}$ can be obtained from the inertia tensor $\mathbf{I}_{s}$ with the main 
axes fixed at $C G$. Without considering the pitch angle, the transformation matrix will be only related to the roll angle $\phi$, i.e.,

$$
\mathbf{R}_{t}=\left[\begin{array}{ccc}
1 & 0 & 0 \\
0 & \cos \phi & -\sin \phi \\
0 & \sin \phi & \cos \phi
\end{array}\right]
$$

To take the rotation and translation of the vehicle body into account, we obtain the inertia tensor $\mathbf{I}_{\mathbf{u}}$ in $O_{u} X_{u} Y_{u} Z_{u}$

$$
\begin{aligned}
\mathbf{I}_{u}=\left[\begin{array}{cc}
I_{x x}+m_{s} h_{s}^{2} & I_{x z} \sin \phi \\
I_{x z} \sin \phi & I_{y y} \cos ^{2} \phi+I_{z z} \sin ^{2} \phi+m_{s} h_{s}^{2} ? \\
-I_{x z} \cos \phi & \left(I_{y y}-I_{z z}\right) \sin \phi \cos \phi \\
-I_{x z} \cos \phi \\
\\
\\
\\
\left(I_{y y}-I_{z z}\right) \sin \phi \cos \phi \\
I_{y y} \sin ^{2} \phi+I_{z z} \cos ^{2} \phi
\end{array}\right]
\end{aligned}
$$

According to the equation (7), relative to the inertia coordinate system $O X Y Z$, the rotational acceleration of sprung mass is computed as follows

$$
\mathbf{M}=\left[\begin{array}{lll}
\mathbf{M}_{1} & \mathbf{M}_{2} & \mathbf{M}_{3}
\end{array}\right]^{T}
$$

where

$$
\begin{gathered}
\mathbf{M}_{1}=\left(I_{x x}+m_{s} h_{s}^{2}\right) \ddot{\phi}-I_{x z} \cos \phi \dot{\gamma} \\
-\left(I_{y y}-I_{z z}\right) \sin \phi \cos \phi \gamma^{2} \\
\mathbf{M}_{2}=I_{x z} \cos \phi \dot{\phi}^{2}+\left(I_{y y}-I_{z z}\right) \cos (2 \phi) \dot{\phi} \gamma \\
-I_{x z} \cos \phi \gamma^{2}+\left(I_{y y}-I_{z z}\right) \sin \phi \cos \phi \dot{\gamma} \\
+\left(I_{x x}+m_{s} h_{s}^{2}\right) \dot{\phi} \gamma+I_{x z} \sin \phi \ddot{\phi}
\end{gathered}
$$

\subsection{DOF Vehicle Model}

It is assumed that forward velocity $v_{x}$ is constant or slowly varying, and a 4DOF vehicle dynamical model can be derived from (11) 


$$
\begin{gathered}
m_{u} a_{y u}+m_{s} a_{y s}=F_{y f}+F_{y r} \\
I_{x z} \sin \phi \dot{\phi}^{2}-I_{x z} \cos \phi \ddot{\phi}+2\left(I_{y y}-I_{z z}\right) \\
\cdot \sin \phi \cos \phi \dot{\phi} \gamma+\left(I_{y y} \sin ^{2} \phi+I_{z z} \cos ^{2} \phi\right) \dot{\gamma} \\
=l_{f} F_{y f}-l_{r} F_{y r}
\end{gathered}
$$

and

$$
\begin{aligned}
& \left(I_{x x}+m_{s} h_{s}^{2}\right) \ddot{\phi}-I_{x z} \cos \phi \dot{\gamma}-\left(I_{y y}-I_{z z}\right) \sin \phi \cos \phi \gamma^{2} \\
& =m_{s} h_{s} a_{y u}+m_{s} g h_{s} \phi-k_{\phi} \phi-c_{\phi} \dot{\phi}+\psi_{3}
\end{aligned}
$$

Using (2), the lateral acceleration $a_{y u}$ is given by

$$
a_{y u}=\dot{v}_{y}+v_{x} \gamma
$$

Assuming the tire model is linear, the lateral forces $F_{y r}$ and $F_{y f}$ of the rear and front axles are given by

$$
\begin{aligned}
F_{y f} & =C_{f} \alpha_{f} \\
F_{y r} & =C_{r} \alpha_{r}
\end{aligned}
$$

where $C_{r}$ and $C_{f}$ denote the effective rear and front cornering stiffness of the rear and front axles. The rear and front tire slip angles $\alpha_{r}$ and $\alpha_{f}$ are as follows

$$
\begin{aligned}
& \alpha_{f}=\delta_{f}-\frac{v_{y}+l_{f} \gamma}{v_{x}}+\kappa_{f} \phi \\
& \alpha_{r}=-\frac{v_{y}-l_{r} \gamma}{v_{x}}+\kappa_{r} \phi
\end{aligned}
$$

Using a state space representation, the vehicle model can be expressed as

$$
E \dot{x}=A_{0} x+B_{0} \delta_{f}+\Psi(x)
$$

where

$$
\begin{aligned}
& x=\left[\begin{array}{llll}
v_{y} & \gamma & \phi & \dot{\phi}
\end{array}\right]^{T}, \\
& B_{0}=\left[\begin{array}{llll}
C_{f} & C_{f} l_{f} & 0 & 0
\end{array}\right]^{T},
\end{aligned}
$$




$$
\begin{aligned}
& A_{0}=\left[\begin{array}{cccc}
a_{11} & a_{12} & a_{13} & 0 \\
a_{21} & a_{22} & a_{23} & 0 \\
0 & 0 & 0 & 1 \\
0 & -m_{s} h_{s} v_{x} & m_{s} g h_{s}-k_{\phi} & -c_{\phi}
\end{array}\right], \\
& E=\left[\begin{array}{cccc}
m & 0 & 0 & -m_{s} h_{s} \\
0 & I_{z z} & 0 & -I_{x z} \\
0 & 0 & 1 & 0 \\
-m_{s} h_{s} & -I_{x z} & 0 & I_{x x}+m_{s} h_{s}^{2}
\end{array}\right], \\
& a_{11}=-\frac{C_{f}+C_{r}}{v_{x}}, a_{12}=\frac{-C_{f} l_{f}+C_{r} l_{r}}{v_{x}}-m v_{x} \text {, } \\
& a_{21}=\frac{-C_{f} l_{f}+C_{r} l_{r}}{v_{x}}, a_{22}=-\frac{C_{f} l_{f}^{2}+C_{r} l_{r}^{2}}{v_{x}}, \\
& a_{13}=C_{f} \kappa_{f}+C_{r} \kappa_{r}, a_{23}=C_{f} \kappa_{f} l_{f}-C_{r} \kappa_{r} l_{r}, \\
& \Psi(x)=\left[\begin{array}{llll}
\psi_{1}(x) & \psi_{2}(x) & 0 & \psi_{4}(x)
\end{array}\right]^{T}, \\
& \psi_{1}(x)=-m_{s} h_{s}\left(\sin \phi \dot{\phi}^{2}+\sin \phi \gamma^{2}\right), \\
& \psi_{2}(x)=-I_{x z} \sin \phi \dot{\phi}^{2}-2\left(I_{y y}-I_{z z}\right) \sin \phi \cos \phi \dot{\phi} \gamma, \\
& \psi_{4}(x)=\left(I_{y y}-I_{z z}\right) \sin \phi \cos \phi \gamma^{2}
\end{aligned}
$$

Assuming that the lateral acceleration and the yaw rate are measurable outputs, it yields

$$
\begin{aligned}
& \dot{x}=A x+B \delta_{f}+L(x) \\
& y=C x+D \delta_{f}
\end{aligned}
$$

where

$$
\begin{gathered}
A=E^{-1} A_{0}, B=E^{-1} B_{0}, L(x)=E^{-1} \Psi(x), \\
C=\left[\begin{array}{cccc}
-\frac{C_{f}+C_{r}}{m v_{x}} & \frac{-C_{f} l_{f}+C_{r} l_{r}}{m v_{x}} & \frac{C_{f} \kappa_{f}+C_{r} \kappa_{r}}{m} & 0 \\
0 & 1 & 0 & 0
\end{array}\right], \\
D=\left[\begin{array}{ll}
\frac{C_{f}}{m} & 0
\end{array}\right]^{T}
\end{gathered}
$$




\section{Observer Design}

Since the roll angle is rarely measured directly due to cost and reliability issues, it is essential to design the asymptotically stable observer based on the available measurements such as the vehicle speed, yaw rate. By utilizing linear matrix inequalities, the observer gain matrix is derived. Assuming the steering angle $\delta_{f}$ can be measured, the following observer is proposed

$$
\begin{aligned}
& \dot{\hat{x}}=A \hat{x}+B \delta_{f}+L(\hat{x})+G(y-\hat{y}) \\
& \hat{y}=C \hat{x}+D \delta_{f}
\end{aligned}
$$

The estimation error dynamics can be evaluated as

$$
\dot{\tilde{x}}=(A-G C) \tilde{x}+L(x)-L(\hat{x})
$$

where $\tilde{x}=x-\hat{x}$.

Proposition: Consider the vehicle system and the proposed observer in (19) and (20), if there exist matrices $P>0$ and $Q$ such that for $\forall j=1, \ldots, n$, and $i=1, \ldots, n$,

$$
\begin{aligned}
& P\left(A+\overline{\mathrm{H}}_{i j}^{\text {max }}\right)+\left(A+\overline{\mathrm{H}}_{i j}^{\max }\right)^{T} P-Q C-C^{T} Q^{T}<0 \\
& P\left(A+\overline{\mathrm{H}}_{i j}^{\text {min }}\right)+\left(A+\overline{\mathrm{H}}_{i j}^{\min }\right)^{T} P-Q C-C^{T} Q^{T}<0
\end{aligned}
$$

then the observer gain matrix $G=P^{-1} Q$ guarantees that the observed error dynamics is asymptotically stable, where $\overline{\mathrm{H}}_{i j}^{\min }=z \mathrm{H}_{i j}^{\min }, \quad \overline{\mathrm{H}}_{i j}^{\max }=z \mathrm{H}_{i j}^{\max }$, $\mathrm{H}_{i j}^{\min }=e_{n}(i) e_{n}^{T}(j) h_{i j}^{\min }$, $\mathrm{H}_{i j}^{\max }=e_{n}(i) e_{n}^{T}(j) h_{i j}^{\max }, h_{i j}^{\min } \leq \min \left(\partial L_{i} / \partial x_{j}\right), h_{i j}^{\max } \geq \max$

$\left(\partial L_{i} / \partial x_{j}\right)$, and $e_{n}$ is the standard basis of the space $R^{n}$, the scaling factor $z=n \times n-l$, and $l$ being the number of elements that $L_{i} / \partial x_{j}$ is equal to zero.

Proof: By using modified mean value theorem [10], the derivative of the $i$ th element of $L(x)$ can be expressed as a convex combination of two values of its derivative

$$
\frac{\partial L_{i}}{\partial x_{j}}(c)=\delta_{i j}^{\max } \frac{\partial L_{i}}{\partial x_{j}}(\Gamma)+\delta_{i j}^{\min } \frac{\partial L_{i}}{\partial x_{j}}(\sigma)
$$

where $\Gamma=\left(\Gamma_{1}, \Gamma_{2}, \ldots, \Gamma_{n}\right)$, and $\sigma=\left(\sigma_{1}, \sigma_{2}, \ldots, \sigma_{n}\right), \Gamma, \sigma$

$\in(a, b) \delta_{i j}^{\max }, \delta_{i j}^{\min }>0, \delta_{i j}^{\max }+\delta_{i j}^{\min }=1$. In view of the vector function mean value theorem [11], it is easy to obtain 


$$
\begin{aligned}
& L(x)-L(\hat{x})=\nabla L(c)(x-\hat{x}) \\
& =\left[\left(\sum_{i, j=1}^{n, n} H_{i j}^{\max } \delta_{i j}^{\max }\right)+\left(\sum_{i, j=1}^{n, n} H_{i j}^{\min } \delta_{i j}^{\min }\right)\right](x-\hat{x})
\end{aligned}
$$

Choose the Lyapunov function candidate as

$$
V(x)=\tilde{x}^{T} P \tilde{x}
$$

and it can be deduced that $\dot{V}<0$, so the estimation error dynamics (21) is asymptotically stable.

\section{Simulations}

To show the effectiveness of the proposed observer, simulation tests are carried out under aggressive driving conditions. Figs. 3 and 4 illustrate the driver inputs during J-turn and fishhook maneuvers. It is known that the maneuvers are utilized by NHTSA to examine the vehicles roll stability. The data of the vehicle system are given as follows: $m=14193 \mathrm{~kg}, m_{s}=12487 \mathrm{~kg}, h_{s}=1.15 \mathrm{~m}, c_{f}=582 * 10^{3} \mathrm{~N} / \mathrm{rad}$, $c_{r}=783 * 10^{3} \mathrm{~N} / \mathrm{rad}, k_{\phi}=380 * 10^{3} \mathrm{~N} / \mathrm{rad}, c_{\phi}=100 * 10^{3} \mathrm{~N} / \mathrm{rad}$, $k_{f}=2060 * 10^{3} \mathrm{~N} / \mathrm{rad}, k_{r}=3337 * 10^{3} \mathrm{~N} / \mathrm{rad}, l_{f}=1.95 \mathrm{~m}, l_{r}=1.54 \mathrm{~m}$, $I_{x x}=24201 \mathrm{kgm}^{2}, I_{x z}=4200 \mathrm{kgm}^{2}, I_{z z}=3491 \mathrm{kgm}^{2}$. For the observer design in these scenarios, we assume that 1) $-45^{\circ} \leq \phi \leq 45^{\circ}$; 2) $-1 \mathrm{rad} / \mathrm{s} \leq \dot{\phi} \leq 1 \mathrm{rad} / \mathrm{s} \quad$ or $\quad-57.3^{\circ} \leq \dot{\phi} \leq 57.3^{\circ} \quad$; $\quad 3$ ) $-15 \mathrm{~m} / \mathrm{s}^{2} \leq a_{y} \leq 15 \mathrm{~m} / \mathrm{s}^{2}$; 4) $\dot{a}_{y}$ is small.

In the first simulation, a step steering input maneuver is performed with initial velocity of $80 \mathrm{~km} / \mathrm{h}$. A steering like step input can realize the transition from straight line to cornering with constant radius. Figs. 5 and 6 illustrate the observed roll rate and roll angle using the proposed observer during J-turn maneuver. It can be shown that the simulated results offer a good match to the real values for this scenario although there exist steering wheel inputs. During the J-turn maneuver, the responses of roll rate and roll angle change sharply between $2 s$ and $3 s$, and there are some oscillations during the initial transients, but finally the estimated values converge to the real values. 


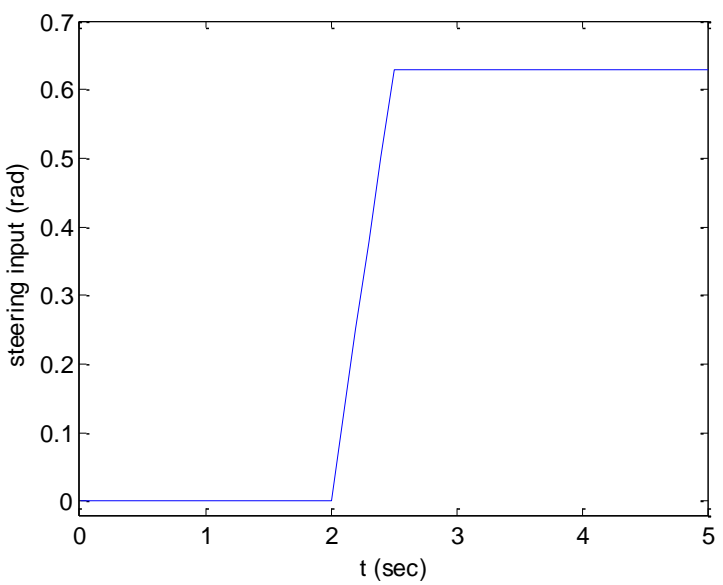

Fig. 3. Steering angles for a J-turn maneuver.

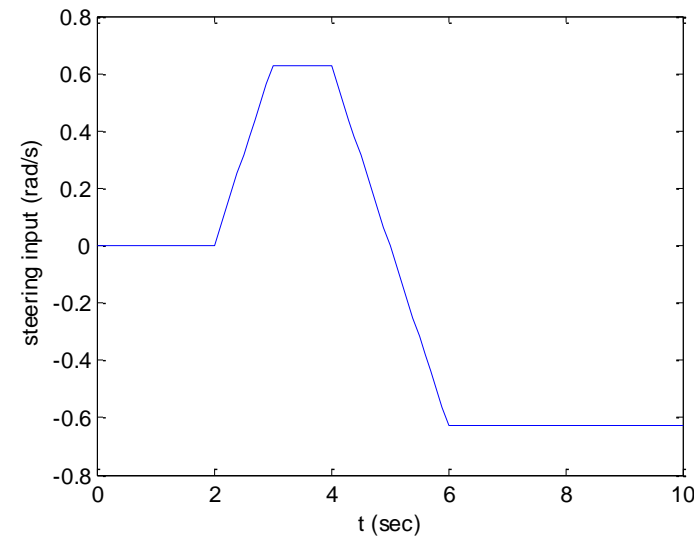

Fig. 4. Steering angles for a fishhook maneuver. 


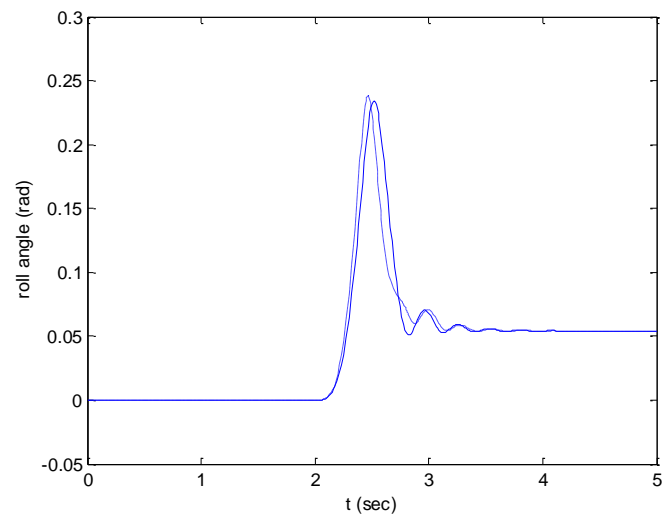

Fig.5. Responses of roll angle durnig a J-turn maneuver (solid-real, dash-dot-estimated).

In the second simulation, a fishhook test is conducted with a vehicle velocity of $80 \mathrm{~km} / \mathrm{h}$. The steering angle input increases at a rate of $36 \mathrm{deg} / \mathrm{s}$ until it reaches $36 \mathrm{deg}$, and the peak value is retained for $1 s$, then a steering angle of opposite direction is conducted at a rate of $36 \mathrm{deg} / \mathrm{s}$ up to $-36 \mathrm{deg}$. Figs. 7 and 8 plot the estimated roll rate and roll angle during the fishhook maneuver. The simulation results show that due to the significantly large steering input, the roll rate and roll angle happen to jump between $2 s$ and $6 s$. However, because the couplings between roll motion, yaw motion, and lateral motion are utilized, the observer provides reliable estimation for this maneuver, even when the vehicle is operating in the nonlinear range.

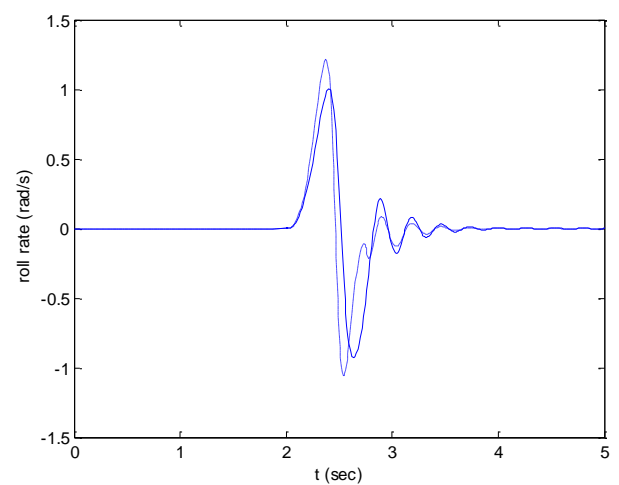

Fig.6. Responses of roll rate during a J-turn maneuver (solid-real, dash-dot-estimated). 


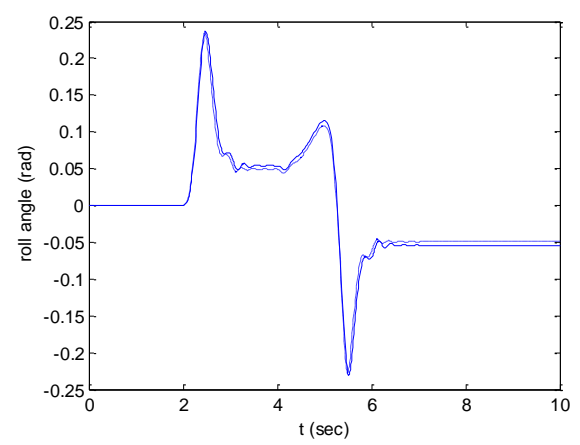

Fig.7. Responses of roll angle durnig a fishhook maneuver (solid-real, dash-dot-estimated)

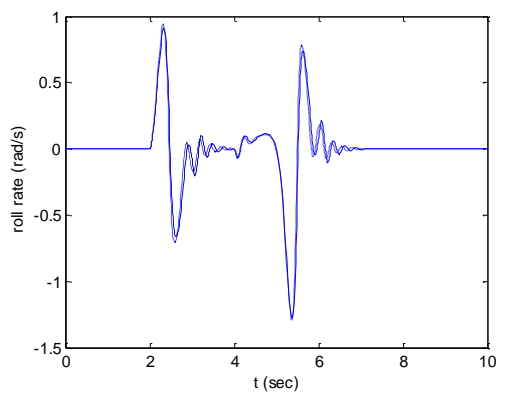

Fig.8. Responses of roll rate during a fishhook maneuver (solid-real, dash-dot-estimated)

\section{Conclusions}

This paper investigates the accurate discovery of the risk of an impending vehicle rollover. To deal with the nonlinear coupling effects between roll dynamics, yaw dynamics, and lateral dynamics, a new vehicle roll dynamics model is developed. On basis of the proposed model, an asymptotically stable observer is presented, and the observer gain matrix is derived by utilizing linear matrix inequalities. Based on the mean value theorem, the nonlinear vehicle dynamics can be expressed as the convex combination of some known matrices, and their coefficients are time-varying. The simulations demonstrate that the proposed nonlinear observer can provide reliable estimation of vehicle states during highly dynamic maneuvers.

\section{Acknowledgment}

This work is supported by the National Natural Science Foundation of China under Grant 61603224 and Grant 61304008. 


\section{References}

[1] National Highway Traffic Safety Administration (NHTSA) [online], available: http://www.nhtsa.dot.gov.

[2] S. Chen, N. Moshchuk, F. Nardi, and J. Ryu, "Vehicle rollover avoidance," IEEE Control Systems Magazine, vol. 30, no. 4, Jul. 2010, pp. 70-85, doi: 10.1109/MCS.2010.937004.

[3] G. Phanomchoeng, R. Rajamani, "New rollover index for the detection of tripped and untripped rollovers," IEEE Transactions on Industrial Electronics, vol. 60, no. 10, Oct. 2013, pp. 4726-4736, doi: 10.1109/TIE.2012.2211312.

[4] L. Xu, H. Eric Tseng, "Robust model-based fault detection for a roll stability control system," IEEE Transactions on Control Systems Technology, vol. 15, no. 3, May 2007, pp. 519-528, doi: 10.1109/TCST.2006.890287.

[5] J. Jung, T. Shim, J. Gertsch, “A vehicle roll-stability indicator incorporating roll-center movements," IEEE Transactions on Vehicular Technology, vol. 58, no. 8, Oct. 2009, pp. 4078-4087, doi: 10.1109/TVT.2009.2021420.

[6] V. Tsourapas, D. Piyabongkarn, A. C. Williams, R. Rajamani, "New method of identifying real-time predictive lateral load transfer ratio for rollover prevention systems," Proceedings of American Control Conference, St. Louis, MO, USA, Jun. 2009, pp. 439-444, doi: 10.1109/ACC.2009.5160061.

[7] S. Solmaz, M. Corless, and R. Shorten, "A methodology for the design of robust rollover prevention controllers for automotive vehicles with active steering," International Journal of Control, vol. 80, no. 11, Oct. 2007, pp. 1763-1779, doi: 10.1080/00207170701473987.

[8] G. Phanomchoeng, and R. Rajamani, "Real-Time estimation of rollover index for tripped rollovers with a novel unknown input nonlinear observer," IEEE/ASME Transactions on Mechatronics, vol. 1, no. 2, Apr. 2014, pp. 743-754, doi: 10.1109/TMECH.2013.2254495.

[9] C. Chen, Y. Jia, M. Shu, Y. Wang, "Hierarchical adaptive pathtracking control for autonomous vehicles," IEEE Transactions on Intelligent Transport Systems, vol. 16, no. 5, Oct. 2015, pp. 2900-2912, doi: 10.1109/TITS.2015.2426211.

[10] K. Eriksson, D. Estep, and C. Johnson, Applied Mathematics: Body and Soul. Berlin: Springer, 2010.

[11] P. K. Sahoo, and T. Riedel, Mean Value Theorems and Functional Equations. Singapore: World Scientific, 1999. 